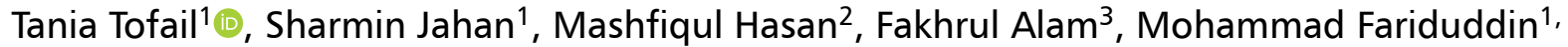
4, Ahmed Abu Saleh ${ }^{1,4}$, Muhammad Abul Hasanat ${ }^{1}$

${ }^{1}$ Department of Endocrinology, Bangabandhu Sheikh Mujib Medical University, Dhaka, Bangladesh

${ }^{2}$ National Institute of Neuroscience, Dhaka, Bangladesh

${ }^{3}$ Abdul Malek Ukil Medical College, Noakhali, Bangladesh

${ }^{4}$ Department of Microbiology, Bangabandhu Sheikh Mujib Medical University, Dhaka, Bangladesh

\title{
Body mass index correlates positively with insulin resistance and secretion but inversely with insulin sensitivity in gestational diabetes
}

\section{ABSTRACT}

Background. To assess fasting C-peptide and insulin indices using homeostatic model assessment (HOMA) in gestational diabetes mellitus (GDM).

Methods. Gestational diabetes mellitus $(n=64$, age: $27.02 \pm 0.53$ years, BMI: $26.3 \pm 0.5 \mathrm{~kg} / \mathrm{m}^{2}$; mean \pm SEM) and normal glucose tolerance NGT $(n=56$, age: $26.11 \pm 0.55$ years, BMI: $24.4 \pm 0.4 \mathrm{~kg} / \mathrm{m}^{2}$; mean \pm SEM) screened according to WHO 2013 criteria. Glucose was measured by oxidase method whereas fasting insulin and C-peptide by ELISA. HOMA-IR, HOMA-B and HOMA-\%S were calculated.

Results. C-peptide was found higher in GDM compared to NGT without any significant difference $(P=0.465)$. Fasting insulin ( $P=0.063)$ and HOMA-IR $(P<0.001)$ were significantly higher while HOMA-B $(P=0.015)$ and HOMA-\%S $(P=0.012)$ were significantly lower in GDM than those of NGT. BMI, bad obstetric history, multiparity and blood glucose were higher $(P \leq 0.05)$ in GDM while age, duration of gestation, family history of DM did not differ ( $P=N S)$. C-peptide (0.185 \pm 0.06 vs $0.331 \pm 0.44 ; P<0.05)$, fasting insulin $(4.88 \pm 0.74$

\section{Address for correspondence:}

Tania Tofail

Department of Endocrinology

Bangabandhu Sheikh Mujib Medical University

Dhaka, Bangladesh

e-mail: tania.ali194@gmail.com

Clinical Diabetology 2021, 10; 3: 270-275

DOI: 10.5603/DK.a2021.0019

Received: 01.01.2021

Accepted: 05.02.2021 vs $10.37 \pm 0.74 ; P<0.01)$ and HOMA-IR $(1.04 \pm 0.14$ vs $2.48 \pm 0.18 ; P<0.011)$ as well as HOMA-B were found lower in GDM having BMI $<23 \mathrm{~kg} / \mathrm{m}^{2}$ than those of GDM with BMI $\geq 23$. Conversely, HOMA-\%S (115.52 \pm 14.63 vs $50.62 \pm 3.39 ; P<0.011$ ) was higher in the subgroup with $\mathrm{BMI}<23$ than the rest. Fasting blood glucose (FBG) $(P<0.01)$ and fasting insulin $(P<0.05)$ were independent predictors for GDM.

Conclusions. Fasting C-peptide did not differ between GDM and NGT. BMI was positively related with resistance and secretion of insulin but inversely with sensitivity. HOMA model analyses revealed decreased insulin sensitivity and secretory capacity in GDM than NGT. (Clin Diabetol 2021; 10; 3: 270-275)

Key words: GDM, Insulin, C-peptide, HOMA IR, HOMA B \& HOMA-\%S

\section{Introduction}

Pregnancy exerts various physiologic adaptations to maintain normal glucose homeostasis. $\beta$-cell needs to compensate for the physiologic insulin resistance by increasing the secretion of insulin [1]. An insufficient compensatory response will lead to gestational diabetes mellitus (GDM). GDM if not treated results in various complications which poses the mother and child at high risk of long-term morbidity [2]. However, many other factors, such as genetic, environmental and autoimmune factors, are thought to be involved in the development of GDM [3]. 
GDM is commonly believed to be primarily associated with insulin resistance (IR). In normal pregnancy (NGT, normal glucose tolerance), dysglycemia does not develop because of increased compensatory insulin secretion. If this compensatory mechanism fails, IR becomes predominant and GDM may develop [4-6]. However, IR alone is not sufficient to cause diabetes [7]. Thus, it is quite reasonable to assume that GDM patients are likely to have both defective insulin secretion and IR. However, observations on insulin secretion and its role in GDM are inconclusive. For instance, while some researchers have shown deficient insulin secretion in response to oral or intravenous glucose [8], others have found comparable or even higher insulin responses in women with GDM $[5,9]$. In Asians, the pancreatic $\beta$-cell mass is relatively smaller compared to Western population, hence the insulin secretion is lower $[8,10]$. Morkrid et al. [10] observed that the South Asian pregnant women lacks $\beta$-cell adaptation compared to Western Europeans.

The physiology of C-peptide makes it appropriate for assessing insulin secretion as it is secreted in equimolar amounts. The best method to determine prehepatic insulin secretion noninvasively is peripheral venous $C$-peptide concentrations [11]. Among various indices for measurement of insulin sensitivity/ resistance, we have employed the homeostasis model assessment (HOMA) model, which correlates well with gold standard clamp techniques [12]. It has been observed earlier that HOMA-B index is lower in GDM women of South Asia $[8,10]$. This study measured fasting Cpeptide as a marker of insulin secretory capacity and compared it with insulin indices using HOMA model between GDM and NGT.

\section{Materials and methods}

Study design and sample

This study enrolled 64 women with GDM (age: $27.02 \pm 4.26$ years, BMl: $26.38 \pm 4.75 \mathrm{~kg} / \mathrm{m}^{2}$; mean \pm SD) and 56 women with NGT (age: $26.11 \pm 4.13$ years, BMI: $24.38 \pm 3.59 \mathrm{~kg} / \mathrm{m}^{2}$; mean \pm SD) screened by 3-sample 75-gm oral glucose tolerance test (OGTT) according to WHO 2013 criteria for GDM. Women irrespective of their duration of gestation with singleton pregnancy attending in the Department of Endocrinology, BSMMU were screened and enrolled consecutively. Women with prior history of DM were excluded from the study.

\section{Clinical, demographic and laboratory data}

It was a prospective observational study done from April, 2018 to February, 2019. The research protocol was approved by the Institutional Review Board (IRB) prior to commencement of this study. After recording
Table 1. WHO 2013 criteria for diagnosis of GDM [13]

\begin{tabular}{lcc}
\hline Time point of OGTT & GDM & DM in pregnancy \\
\hline 0-hour PG & $5.1-6.9 \mathrm{mmol} / \mathrm{L}$ & $\geq 7.0 \mathrm{mmol} / \mathrm{L}$ \\
1-hour PG & $\geq 10.0 \mathrm{mmol} / \mathrm{L}$ & - \\
2-hour PG & $8.5-11.0 \mathrm{mmol} / \mathrm{L}$ & $\geq 11.1 \mathrm{mmol} / \mathrm{L}$ \\
\hline
\end{tabular}

DM - diabetes mellitus; GDM - gestational diabetes mellitus; OGTT — oral glucose tolerance test; PG — plasma glucose

clinical data in the case record form, OGTT was performed following an overnight fast. Study subjects were included as GDM or NGT on the basis of WHO 2013 diagnostic criteria. Fasting venous blood $(6 \mathrm{ml})$ was collected for measurement of C-peptide and insulin. Serum was preserved at $-80^{\circ} \mathrm{C}$ until assay.

\section{Oral glucose tolerance test (OGTT)}

WHO (2013) recommend a 75g OGTT and the diagnosis of GDM are made irrespective of weeks of gestation when any one or more of the following plasma glucose (PG) criteria are fulfilled (Table 1).

\section{Analytic method}

Plasma glucose was analyzed by glucose oxidase method by Dimension EXL 200 Integrated Chemistry System (Siemens, Germany) on the same day of collection. Serum insulin levels were measured by chemiluminescent immunoassay method using Access Immunoassay System (REF: 33410), Beckman Coulter, Inc., USA. Quantitative determination of serum C-peptide levels was done by two-site chemiluminnescent immunometric assay using Immulite 2000 System Analyzers (Cat. No. L2KPEP2), Siemens, Inc., Germany. The coefficient variances (CV) for glucose were $2.03 \%$ for low level values and $2.08 \%$ for high values level where intra assay CV for insulin was $2.54 \%$. Intra assay CV for C-peptide was $2.34 \%$.

\section{Assessment of insulin secretion}

nd sensitivity index

Insulin resistance and secretion were calculated by the equations of HOMA model described by Matthews et al. [12].

\section{Statistical analysis}

Data were analyzed using SPSS version 23.0. Qualitative data were expressed as frequencies or percentages. Assessment of normality of quantitative data was done by Shapiro-Wilk test. All the quantitative data were found normally distributed and expressed as mean \pm SEM. Among subgroups made on the basis of clinical and metabolic (hormonal and derived insulin 
Table 2. Demographic characteristics of studied subjects

\begin{tabular}{|c|c|c|c|}
\hline \multirow[t]{2}{*}{ Variable } & \multicolumn{2}{|c|}{ Population } & \multirow[t]{2}{*}{$p$} \\
\hline & GDM $(n=64)$ & NGT $(n=56)$ & \\
\hline Age [years] ${ }^{a}$ & $27.02 \pm 0.53$ & $26.11 \pm 0.55$ & 0.239 \\
\hline Body weight $[\mathrm{kg}]^{\mathrm{a}}$ & $63.11 \pm 1.47$ & $58.83 \pm 1.23$ & 0.03 \\
\hline BMI $\left[\mathrm{kg} / \mathrm{m}^{2}\right]^{\mathrm{a}}$ & $26.3 \pm 0.5$ & $24.42 \pm 0.4$ & 0.011 \\
\hline Weeks of gestation at admission [weeks] ${ }^{a}$ & $23.08 \pm 1.23$ & $20.50 \pm 1.24$ & 0.146 \\
\hline $\mathrm{SBP}[\mathrm{mm} \mathrm{Hg}]^{\mathrm{a}}$ & $103.98 \pm 1.64$ & $99.91 \pm 1.46$ & 0.070 \\
\hline $\mathrm{DBP}[\mathrm{mm} \mathrm{Hg}]^{\mathrm{a}}$ & $66.56 \pm 0.98$ & $65.09 \pm 0.92$ & 0.279 \\
\hline Multiparity & $41(64.06 \%)$ & $22(39.29 \%)$ & $0.007^{*}$ \\
\hline Bad obstetric history ${ }^{b}$ & $16(25.0 \%)$ & $7(12.5 \%)$ & $0.055^{*}$ \\
\hline Family history of $\mathrm{DM}^{\mathrm{b}}$ & $18(28.13 \%)$ & $20(35.1 \%)$ & $0.373^{*}$ \\
\hline
\end{tabular}

$\mathrm{BMI}$ - body mass index; DBP — diastolic blood pressure; $\mathrm{FH}$ - family history of DM in $1^{\text {st }}$ degree relatives; GDM - gestational diabetes mellitus;

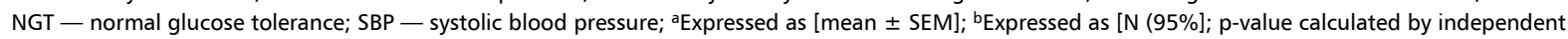
$t$-test and *expressed as $\mathrm{p}$ value calculated Chi-square test

indices) findings, comparison was done by chi-square test and independent sample t-test. Correlation among BMI, C-peptide, insulin indices and derived variables were calculated by Pearson's correlation test. Logistic regression analysis was done to determine the predictive association of BMI and insulin indices in pregnant women with or without GDM. P value $<0.05$ was considered as statistically significant.

\section{Results}

In this study we investigated the secretory capacity of islet cells by measurement of fasting C-peptide in GDM as well as NGT and compared between these two groups. Also fasting glucose and insulin indices were measured and compared.

Table 2 depicts demographic variables (mean + SEM or\%). Bodyweight $(63.11 \pm 1.47$ vs $58.83 \pm 1.23 ; \mathrm{P}=0.03)$; BMI $\left(26.3 \pm 0.5\right.$ vs $\left.24.4 \pm 0.4 \mathrm{~kg} / \mathrm{m}^{2}, \mathrm{P}=0.011\right)$, multiparity (41 vs $22, \mathrm{P}=0.007$ ) and bad obstetric history (16 vs 7, P = 0.055) were statistically and significantly higher in the GDM group than in those with NGT while other variables, i.e., age ( $27.02 \pm 0.53$ vs $26.11 \pm 0.55$ years, $P=0.239)$, gestational age $(23.08 \pm 1.23$ vs $20.50 \pm$ \pm 1.24 weeks, $P=0.146)$, family history of diabetes (18 vs $20, P=0.373$ ), didn't show any remarkable difference.

As displayed in Table 3, all the glucose values were significantly higher in GDM than in NGT (FBG: $5.27 \pm$ \pm 0.08 vs $4.44 \pm 0.05, \mathrm{P}<0.001 ; 01 \mathrm{hr}$ blood glucose: $9.93 \pm 0.22$ vs $7.70 \pm 0.15, \mathrm{P}<0.001 ; 2 \mathrm{hr}$ blood glucose: $8.43 \pm 0.21$ vs $6.53 \pm 0.15, \mathrm{P}<0.001)$. Fasting insulin (9.431 \pm 0.63 vs $7.557 \pm 0.72, P$ 0.063) and HOMA IR (2.24 \pm 0.17 vs $1.49 \pm 0.13$, P 0.001) were significantly higher while HOMA B (120.56 \pm 10.42 vs $180.64 \pm 22.81, \mathrm{P} 0.015)$ and HOMA-\%S (61.76 \pm
4.82 vs $122.69 \pm 24.88$, P 0.012 ) were significantly lower in GDM than in NGT. C-peptide did not show any significant difference between the two groups, but was higher in the GDM than in NGT $(0.34 \pm 0.07$ vs $0.26 \pm 0.06, P=0.465$ ).

Table 4 shows the comparisons of various facets of insulin indices between BMI subgroups of GDM divided by the cutoff at $23 \mathrm{~kg} / \mathrm{m}^{2}$. C-peptide $(0.185 \pm 0.06$ vs $0.331 \pm 0.44 ; \mathrm{P}<0.05)$, fasting insulin $(4.88 \pm 0.74$ vs $10.37 \pm 0.74 ; P<0.01)$ and HOMA IR $(1.04 \pm 0.14$ vs $2.48 \pm 0.18 ; \mathrm{P}<0.011)$ as well as HOMA B were found lower in the subgroup having $B M I<23 \mathrm{~kg} / \mathrm{m} 2$ than in those with $\mathrm{BMI} \geq 23$. Conversely, HOMA-\%S (115.52 \pm 14.63 vs $50.62 \pm 3.39 ; P<0.011$ ) was higher in the group with $\mathrm{BMI}<23$ than in women with $\mathrm{BMI} \geq 23$.

Table 5 illustrates the predictability of different variables for development of GDM. Only FBG $(P<0.01)$ and fasting insulin $(P<0.05)$ showed significant predictive association. However BMI, family history of DM and bad obstetric history $(\mathrm{BOH})(\mathrm{P}<0.07)$ also had a near-significant level of predictive association.

\section{Discussion}

We designed this study to explore the relationship of insulin secretion, resistance and sensitivity in GDM. It clearly demonstrated that fasting C-peptide indicating insulin secretion does not differ significantly between GDM and NGT despite a higher corresponding blood glucose and higher BMI in the former group. However, insulin secretion was found higher in GDM, with higher $\mathrm{BMI}$ reflecting altered potential of insulin secretion and activity in GDM irrespective of glycemic status and obesity. GDM patients with higher BMI showed increased insulin resistance and secretion, but decreased insulin sensitivity, whereas lean GDM mothers had decreased 
Table 3. Fasting glucose, insulin, C-peptide and insulin indices in GDM and NGT

\begin{tabular}{|c|c|c|c|}
\hline \multirow[t]{2}{*}{ Variable } & \multicolumn{2}{|c|}{ Population } & \multirow[t]{2}{*}{$\mathbf{P}$} \\
\hline & GDM & NGT & \\
\hline $\mathrm{FBG}[\mathrm{mmol} / \mathrm{mL}]^{\mathrm{a}}$ & $5.27 \pm 0.08$ & $4.44 \pm 0.05$ & $<0.001$ \\
\hline $1 \mathrm{hr} \mathrm{BG}[\mathrm{mmol} / \mathrm{mL}]^{\mathrm{a}}$ & $9.93 \pm 0.22$ & $7.70 \pm 0.15$ & $<0.001$ \\
\hline $2 \mathrm{hr} \mathrm{BG}[\mathrm{mmol} / \mathrm{mL}]^{\mathrm{a}}$ & $8.43 \pm 0.21$ & $6.53 \pm 0.15$ & $<0.001$ \\
\hline Fasting insulin $[\mu \mathrm{IU} / \mathrm{mL}]^{a}$ & $9.431 \pm 0.632$ & $7.557 \pm 0.728$ & 0.063 \\
\hline C-peptide $[\mathrm{ng} / \mathrm{mL}]^{\mathrm{a}}$ & $0.34 \pm 0.07$ & $0.26 \pm 0.06$ & 0.465 \\
\hline HOMA IR & $2.24 \pm 0.17$ & $1.49 \pm 0.13$ & $<0.001$ \\
\hline HOMA Ba & $120.56 \pm 10.42$ & $180.64 \pm 22.81$ & 0.015 \\
\hline HOMA-\%S & $61.76 \pm 4.82$ & $122.69 \pm 24.8$ & 0.012 \\
\hline
\end{tabular}

GDM - gestational diabetes mellitus; NGT - normal glucose tolerance; HOMA-IR - homeostasis model assessment of insulin resistance; HOMA-B - homeostasis model assessment of $\beta$-cell function; HOMA-\%S - homeostasis model assessment of insulin sensitivity Number of subjects for C-peptide: $\mathrm{GDM}=36, \mathrm{NGT}=26$

${ }^{a}$ Expressed as [mean $\pm \mathrm{SEM}$ ]; $\mathrm{p}$-values calculated by independent samples t-test

Table 4. Insulin indices in GDM according to BMI categories $\left(23 \mathrm{~kg} / \mathrm{m}^{2}\right)$

\begin{tabular}{lccc}
\hline Variables & \multicolumn{2}{c}{ Groups } & P \\
\cline { 2 - 4 } & $\mathbf{B M I}<\mathbf{2 3} \mathbf{~ k g} / \mathbf{m}^{\mathbf{2}}(\mathbf{n}=\mathbf{9})$ & $\mathbf{B M I} \geq \mathbf{2 3} \mathbf{~ k g} / \mathbf{m}^{\mathbf{2}} \mathbf{( n = 5 3 )}$ \\
\hline C-peptide $[\mathrm{ng} / \mathrm{mL}]$ & $0.185 \pm 0.06$ & $0.331 \pm 0.44$ & $\mathbf{0 . 0 2 8}$ \\
Fasting insulin $[\mu \mathrm{IU} / \mathrm{mL}]$ & $4.88 \pm 0.74$ & $10.37 \pm 0.74$ & $<0.001$ \\
HOMA-IR & $1.04 \pm 0.14$ & $2.48 \pm 0.18$ & $<0.011$ \\
HOMA-B & $89.04 \pm 22.01$ & $127.09 \pm 11.61$ & 0.146 \\
HOMA-\%S & $115.52 \pm 14.63$ & $50.62 \pm 3.39$ & $<0.011$ \\
\hline
\end{tabular}

Data were expressed as mean \pm SD

Comparison between groups done by Student t-test

Table 5. Binary logistic regression

\begin{tabular}{lccc}
\hline Variables in the equation & B & SE & P \\
\hline Age & -0.034 & 0.115 & 0.765 \\
BMI $\left[\mathrm{kg} / \mathrm{m}^{2}\right]$ & 0.314 & 0.173 & 0.070 \\
Gestational week & -0.044 & 0.045 & 0.328 \\
Systolic BP & -0.018 & 0.035 & 0.601 \\
FBG & -5.300 & 1.587 & $<0.001$ \\
Fasting Insulin & -0.457 & 0.183 & 0.013 \\
C-peptide & -0.736 & 1.204 & 0.541 \\
Family history of DM & -2.079 & 1.099 & 0.059 \\
Bad obstetric history & 2.702 & 1.479 & 0.068 \\
Multiparity & -2.093 & 1.341 & 0.118 \\
Constant & 26.077 & 9.448 & 0.006 \\
\hline
\end{tabular}

GDM population is distinct from NGT population $(P=0.006)$

resistance and secretion, but significantly increased sensitivity of insulin. Therefore other possible factors need investigation to explore the phenomenon of GDM in lean mothers.
Characteristics of the subjects were statistically similar except for BMI between the groups of GDM and NGT. A remarkable observation of this study is that there was no significant difference in fasting C-peptide levels between GDM and NGT. This finding is consistent with the observations by some other investigators where basal C-peptide did not vary significantly between the two groups [14]. Higher insulin secretion and higher insulin resistance along with low sensitivity was observed in GDM patients compared with women with NGT. However, insulin resistance and secretion were higher and insulin sensitivity was lower in relatively obese mothers than their lean counterparts in the GDM group. On the other hand, in the face of lower BMI, lean GDM mothers had lower insulin resistance and insulin secretion but higher insulin sensitivity. Nevertheless, both the groups have expressed GDM. On the one hand, GDM patients had higher insulin secretion and resistance, but higher C-peptide than those with NGT; on the other hand, lean GDM mothers had low C-peptide, low insulin resistance, but higher insulin sensitivity than obese GDM patients. Therefore, unless other factors are involved, it is unlikely that GDM mothers of both 
the groups would develop glycemic intolerance. It can also be mentioned that higher glucose level in GDM did not proportionately influence the secretion of insulin or appeared the outcome of insulin resistance in GDM when compared to NGT mothers. Both groups had significantly higher BMI. This also does not seem to be merely attributable to only higher BMI in GDM; because insulin sensitivity as well as insulin resistance and secretion significantly differed between lean and obese GDM mothers which diminished the possibility of stronger influence of glucose and BMI over the aberration of insulin activity in GDM. Hence, it is again pertinent to think that other factors, such as environmental and hormonal factors, genetic aberrance and ethnic influences, are important in our population. Apropos of this, our group has observed under pilot scheme that there is genetic polymorphism of TCF7L2 in our GDM mothers which is higher in frequency in lean and young mothers. Some other studies in broader scale have also observed genetic aberration among GDM mothers of Southeast Asian population [15]. The possibility that there is a shared gene causing glycemic intolerance expressed as GDM during pregnancy among the young and low BMI mothers should be investigated. We are planning to analyze the frequency of genetic alteration to find potentially relevant associations.

$\mathrm{BMI}$ has a strong influence on IR in GDM. Consistent with another study, GDM women with higher BMI $(\geq 23$ $\mathrm{kg} / \mathrm{m}^{2}$ ) had decreased insulin sensitivity and significantly increased insulin resistance [16]. Interestingly, in women with lower BMI $\left(<23 \mathrm{~kg} / \mathrm{m}^{2}\right)$, there was statistically significant and markedly decreased C-peptide concentration. This implies GDM development in lean women might be primarily related to poor insulin secretory capacity. Considering the similarity of GDM pathology with the pathogenesis of type 2 diabetes, it has been postulated that lower insulin secretory capacity is an important risk factor for development of type 2 diabetes in non-obese Asians [8]. This study clearly illustrates the role of $\beta$-cell dysfunction in lean GDM women. It might indicate that other factors are important for high prevalence of GDM. As mentioned above, our GDM Study group has also observed some genetic aberrations in GDM and preponderance in lean and young GDM.

IR and impaired $\beta$-cell function are the central pathogenic mechanisms for developing GDM [1]. Higher IR in GDM was also reflected in a recent work of our GDM study group, BSMMU [17]. Nevertheless, insulin secretory capacity measured by HOMA-B in this study was much lower than the findings of other authors [18]. This variance may also explain the onset of GDM despite lower IR even in non-obese younger mothers of our population.
This study evaluated insulin resistance with fasting insulin and HOMA-IR values and found those values to be significantly higher in GDM than in NGT. Insulin secretory index HOMA-B was significantly lower in GDM than NGT mothers, along with lower insulin sensitivity as measured by HOMA-\%S. Some investigators suggested that GDM is a defect of islet beta cell functions and failure to increase insulin secretion in response to increased IR during pregnancy [19]. Present findings are consistent with their reports, that GDM is due to both reduced insulin secretion and enhanced IR $[6,19]$. We have measured exogenus glucose disposal using sequential insulin infusions with the euglycemic glucose clamp technique and erythrocyte insulin binding. It is worth mentioning that high fasting serum insulin levels in GDM reported in our study are also in agreement with previous studies [1, 8]. Higher fasting insulin level is known to be an established indicator of IR [20].

To summarize, fasting C-peptide does not differ significantly between women with GDM and NGT. There is insulin resistance in GDM expressed in the form of higher insulin than that in NGT. This is also true even when there is undetectable secretion of C-peptide by pancreas in the face of abnormally higher glucose in GDM. In present study, HOMA model analyses revealed decreased insulin sensitivity and secretory capacity in GDM compared with that observed in NGT, and the former was more prominent in overweight GDM whereas the latter in lean or normal-weight women with GDM. Present study investigated C-peptide and fasting insulin levels and was unable to take into account the effect of other factors associated with GDM. Pre-pregnancy BMI could not be used for comparison between the study subjects. Owing to the cross-sectional nature no causal inference could be drawn.

\section{Acknowledgements}

We would like to thank the department of Microbiology, BSMMU for their technical support.

\section{Conflict of interest}

None declared.

\section{REFERENCES}

1. Retnakaran R, Ye C, Kramer CK, et al. Evaluation of circulating determinants of beta-cell function in women with and without gestational diabetes. J Clin Endocrinol Metab. 2016; 101(7): 26832691, doi: 10.1210/jc.2016-1402, indexed in Pubmed: 27023450.

2. Hyperglycemia and Adverse Pregnancy Outcome (HAPO) Study: Associations With Neonatal Anthropometrics. Diabetes. 2008; 58(2): 453-459, doi: 10.2337/db08-1112.

3. Ferrara A, Ehrlich SF. Strategies for diabetes prevention before and after pregnancy in women with GDM. Curr Diabetes Rev. 2011; 7(2): 75-83, doi: 10.2174/157339911794940738, indexed in Pubmed: 21222619. 
4. Catalano PM, Drago NM, Amini SB. Longitudinal changes in pancreatic beta-cell function and metabolic clearance rate of insulin in pregnant women with normal and abnormal glucose tolerance. Diabetes Care. 1998; 21(3): 403-408, doi: 10.2337/ diacare.21.3.403, indexed in Pubmed: 9540023.

5. Kautzky-Willer A, Prager R, WaldhausI W, et al. Pronounced insulin resistance and inadequate beta-cell secretion characterize lean gestational diabetes during and after pregnancy. Diabetes Care. 1997; 20(11): 1717-1723, doi: 10.2337/diacare.20.11.1717, indexed in Pubmed: 9353615.

6. Ryan EA, O'Sullivan MJ, Skyler JS. Insulin action during pregnancy. Studies with the euglycemic clamp technique. Diabetes. 1985; 34(4): 380-389, doi: 10.2337/diab.34.4.380, indexed in Pubmed: 3882502.

7. Shimoyama R, Ray TK, Savage $C R$, et al. In vivo and in vitro effects of antiinsulin receptor antibodies. J Clin Endocrinol Metab. 1984; 59(5): 916-923, doi: 10.1210/jcem-59-5-916, indexed in Pubmed: 6480811.

8. Yang SJ, Kim TN, Baik SH, et al. Insulin secretion and insulin resistance in Korean women with gestational diabetes mellitus and impaired glucose tolerance. Korean J Intern Med. 2013; 28(3): 306-313, doi: 10.3904/kjim.2013.28.3.306, indexed in Pubmed: 23682224.

9. Catalano PM, Huston L, Amini SB, et al. Longitudinal changes in glucose metabolism during pregnancy in obese women with normal glucose tolerance and gestational diabetes mellitus. Am J Obstet Gynecol. 1999; 180(4): 903-916, doi: 10.1016/s00029378(99)70662-9, indexed in Pubmed: 10203659.

10. Mørkrid K, Jenum AK, Sletner $L$, et al. Failure to increase insulin secretory capacity during pregnancy-induced insulin resistance is associated with ethnicity and gestational diabetes. Eur J Endocrinol. 2012; 167(4): 579-588, doi: 10.1530/EJE-12-0452, indexed in Pubmed: 22889687.

11. Jones AG, Hattersley AT. The clinical utility of C-peptide measurement in the care of patients with diabetes. Diabet Med. 2013; 30(7): 803-817, doi: 10.1111/dme.12159, indexed in Pubmed: 23413806.
12. Matthews DR, Hosker JP, Rudenski AS, et al. Homeostasis model assessment: insulin resistance and beta-cell function from fasting plasma glucose and insulin concentrations in man. Diabetologia. 1985; 28(7): 412-419, doi: 10.1007/BF00280883, indexed in Pubmed: 3899825.

13. WHO | Diagnostic criteria and classification of hyperglycaemia first detected in pregnancy. WHO 2013

14. Homko C, Sivan E, Chen $X$, et al. Insulin secretion during and after pregnancy in patients with gestational diabetes mellitus. J Clin Endocrinol Metab. 2001; 86(2): 568-573, doi: 10.1210/ jcem.86.2.7137, indexed in Pubmed: 11158010.

15. MA H. TCF7L2 gene rs7903146 polymorphism may confer expression of gestational diabetes mellitus in relatively young and lean mothers. Diabetes \& Obesity International Journal. 2016; 1(6), doi: $10.23880 /$ doij-16000132.

16. Genova M, Todorova-Ananieva K, Tzatchev K. Impact of body mass index on insulin sensitivity/ resistance in pregnant women with and without gestational diabetes mellitus. vol XL, 2013.

17. Fakhrul-Alam M, Rakibul-Hasan $M$, Farid-Uddin $M$, et al. Insulin secretory defect may be the major determinant of GDM in Iean mothers. J Clin Transl Endocrinol. 2020; 20: 100226, doi: 10.1016/j.jcte.2020.100226, indexed in Pubmed: 32382513.

18. Sokup A, Ruszkowska-Ciastek B, Góralczyk K, et al. Insulin resistance as estimated by the homeostatic method at diagnosis of gestational diabetes: estimation of disease severity and therapeutic needs in a population-based study. BMC Endocr Disord. 2013; 13: 21, doi: 10.1186/1472-6823-13-21, indexed in Pubmed: 23819910.

19. Wei J, Gao J, Cheng J. Gestational diabetes mellitus and impaired glucose tolerance pregnant women. Pak J Med Sci. 2014; 30(6): 1203-1208, doi: 10.12669/pjms.306.5755, indexed in Pubmed: 25674108.

20. Genova MP, Todorova-Ananieva K, Atanasova B, et al. Assessment of beta-cell function during pregnancy and after delivery. Acta Medica Bulgarica. 2014; 41(1): 5-12, doi: 10.2478/amb2014-0001 